\title{
62. COMETS AND PROBLEMS OF NUMERICAL CELESTIAL MECHANICS
}

\author{
S. K. VSEKHSVYATSKI J \\ Astronomical Observatory, Kiev University, Kiev, U.S.S.R.
}

\begin{abstract}
A number of problems concerning the motions and orbital statistics of comets are suggested for numerical discussion.
\end{abstract}

Laplace (1805) was the first to consider cometary problems in a statistical manner. He established the probability for the appearance of comets moving in hyperbolic and almost parabolic orbits on the assumption that these comets came from interstellar space. It was subsequently demonstrated that this assumption was invalid. The lack of observed initial hyperbolic orbits serves as indisputable proof that comets originate in the solar system.

Statistical analysis of the directions of aphelion of the known comets leads us to the same conclusion. The pioneering work of Carrington and Svedstrup has been carried on with different material and by different methods by Fabry, Holetschek, Pickering, Eddington, Oppenheim, Bourgeois and Cox, Witkowski and Hurnik. In all these studies there was found to be no predominance of aphelia in the hemisphere including the apex of the Sun's way. However, there still appear in the literature the results of attempts to apply the idea of interstellar origin on account of the fact that the aphelion distribution hints somewhat at a connection with the structure of the Galaxy, or by calculating the directions of the apices of two comet streams (Witkowski, 1965). If one recalls that the observed comets have relative velocities of less than $0.1 \mathrm{~km} \mathrm{~s}^{-1}$ at the boundary of the Sun's sphere of action, and that the supposed comet streams could not bear any relation to interstellar regions - for which relative velocities would be expressed in kilometres and tens of kilometres per second - it becomes evident that there are no grounds for interpreting the results in such a manner. At relative velocities of less than $0.1 \mathrm{~km} \mathrm{~s}^{-1}$ (which is very small compared to the motion of the Sun in the Galaxy), cometary fragments (even those with periods of $10^{9} \mathrm{yr}$ ) must have come in from distances not greater than a few tens of parsecs, and consequently they could not reflect the peculiarities of the structure of the Galaxy. There exist considerable deviations from a uniform distribution, but these can be assigned to the effects of perturbations by neighbouring stars (Vsekhsvyatskij, 1967). At present, calculation of the effects of individual stars on an individual comet is merely an academic matter, although it may not be impossible to make such calculations in the future.

We may make important conclusions from calculations of original and future orbits of comets. The results by Thraen, Fayet and Strömgren concerning the original elliptical character of the orbits of the majority of comets observed to have osculating hyperbolic orbits are confirmed in the recent summaries by I. Galibina and Z. Sekanina. This also very definitely shows that all the observed comets belong to the solar system, 
although it is true that we have to assume that beyond the bounds of the observed arc the motion of a comet is not influenced by internal or external forces other than gravitational attractions by the known planets.

The nongravitational effects are one of the most basic problems of cometary astronomy and cosmogony. Long ago, study of the motion of Encke's Comet, and later of the motions of other short-period comets, made it possible to determine cases of accelerations or decelerations unexplained by the influence of ordinary perturbations. I explained these effects $40 \mathrm{yr}$ ago on the basis of the eruption theory as a consequence of relative youth and physical instability of a comet (Vsekhsvyatskij, 1931, 1933, 1950). An important contribution to the problem was made by F. L. Whipple and his disciples, in which the idea was advanced that the nucleus of a comet is basically composed of ice.

From the results by Whipple (1950, 1951), Hamid and Whipple (1953), Marsden (1968, 1969), and Sekanina (1968a, 1968b) concerning the nongravitational effects on cometary motion, as well as from the rich collection of numerical theories of the motions of numerous short-period comets, one can make two fundamental conclusions:

(1) nongravitational secular accelerations or decelerations in the motions of comets are appreciable in magnitude, and for comets that make close approaches to Jupiter they lead to considerable deviations from purely gravitational theory after only a small number of revolutions;

(2) nongravitational effects apparently decrease as a comet ages and are on the whole determined by explosive processes in the cometary nucleus. It follows that extrapolation of the motion of a comet over long intervals of time can have little meaning. This is especially true of calculations back into the past, when the nongravitational effects would have been larger. One has thus every reason to consider that the calculations on comets such as Lexell, Wolf, Brorsen, Oterma and others do not point to these comets leaving the Jupiter system before the time of their close approaches before discovery.

Therefore, to elucidate the real value of these extrapolations it would be desirable to recalculate the motions of these comets, varying the initial data (within the bounds of probable error and considering also the nongravitational effects) and comparing the results in order to determine the number of revolutions over which the extrapolations may be considered reliable. This is a particularly important problem. Unless one continues investigations similar to that by Leverrier (1857) on $\mathrm{P} /$ Lexell with due consideration of nongravitational effects as well, one cannot believe that the results are of real significance. The same is true of the calculations of several short-period comets over the interval 1660-2060 (Kazimirchak-Polonskaya, 1967), where extrapolation of the motion of $\mathrm{P} /$ Kearns-Kwee seems to result in a strongly hyperbolic orbit of a type never observed before. It follows from elementary considerations that the discovery of a sharply hyperbolic comet with perihelion distance less than $5 \mathrm{AU}$ is many thousand times more probable than a hyperbolic orbit resulting from a close approach to Jupiter calculated for 1855. The fact that no such comet has ever been seen shows the unreliability of the extrapolated results. 
In recent years detailed and valuable work on conventional orbital statistics has been published by Everhart $(1967,1968,1969)$. Using much more numerical material, he has fully confirmed the correctness of the conclusions made earlier by $\mathrm{H}$. A. Newton, those obtained in investigations in $\mathrm{Kiev}$, as well as preliminary calculations by A. van Woerkom, Z. Sekanina and others on the accumulative effects of small perturbations. Everhart has shown that short-period comets (i.e., the newest and most rapidly disintegrating objects) could not be formed as the result of diffusion or capture from the system of long-period comets, confirming conclusions made long ago by R. A. Proctor, A. C. D. Crommelin and myself.

It is also important to continue statistical calculations on the eruptive theory. We have obtained remarkable agreement between the theoretical and observed distributions of elements of cometary orbits (Vsekhsvyatskij and Meshcheryakova-Babich, 1957), but it is of some interest to expand these calculations to all the giant planets, considering both the satellites and the planets themselves to be possible sources of the comets and varying the initial conditions.

Statistical analysis of the effects of stars passing through the hypothetical Oort comet cloud and study of the stability of motion near the boundaries of the Sun's sphere of action provides an independent means for interpreting the cometary problem. The radius of the Sun's sphere of action in the galactic field is some $70000 \mathrm{AU}$.

Among other important numerical cometary problems we can mention the construction of theories of motion for 'new' (i.e., rapidly disintegrating) short-period comets which passed close to Jupiter shortly before discovery; if one assumes that the minimum separation was close to zero and then tries to obtain an orbit representing all the observations at the discovery apparition, new data may be obtained concerning the evolution of the nongravitational effects.

It is also desirable to compare the motions of comets in their preperihelion and postperihelion branches. This will not only clarify the nongravitational effects but will make it possible to study the effects of outbursts in cometary brightness, nuclear splitting, and so on, with a view to obtaining estimates of the masses of cometary nuclei. A thorough investigation of the motions of particularly interesting comets, coupled with consideration of their physical activity, can give new information about the influence of the solar wind, Poynting-Robertson effect and magnetic fields on the motions of comets and meteoroids.

\section{References}

Everhart, E.: 1967, Astron. J. 72, 716, 1002.

Everhart, E.: 1968, Astron. J. 73, 1039.

Everhart, E.: 1969, Astron. J. 74, 735.

Hamid, S. E. and Whipple, F. L.: 1953, Astron. J. 58, 100.

Kazimirchak-Polonskaya, E. I.: 1967, Trudy Inst. Teor. Astron. 12; Astron. Zh. 44, 439.

Laplace, P.: 1805, Connais. Temps. 1806.

Leverrier, U. J. J.: 1857, Ann. Obs. Paris Mem. 3, 203.

Marsden, B. G.: 1968, Astron. J. 73, 367.

Marsden, B. G.: 1969, Astron. J. 74, 720.

Sekanina, Z.: 1968a, Bull. Astron. Inst. Czech. 19, 351. 
Sekanina, Z.: 1968b, Probl. Kosmich. Fiz. No. 3.

Vsekhsvyatskij, S. K.: 1931, Astron. Nachr. 243, 286.

Vsekhsvyatskij, S. K.: 1933, Astron. Zh. 10, 18.

Vsekhsvyatskij, S. K.: 1950, Astron. Zh. 27, 23.

Vsekhsvyatskij, S. K.: 1967, Priroda $i$ Proiskhozhdenie Komet $i$ Meteornogo Veshchestva, Prosveshchenie, Moscow, p. 150.

Vsekhsvyatskij, S. K. and Meshcheryakova-Babich, O. I.: 1957, Astron. Zh. 34, 568.

Whipple, F. L.: 1950, Astrophys. J. 111, 375

Whipple, F. L.: 1951, Astrophys. J. 113, 464.

Witkowski, J.: 1965, Acta Astron. 15, 273. 